\title{
Quantum-Chemical and Molecular Dynamics Investigations of Magnesium Chloride Complexes in Dimethoxyethane Solutions
}

Piotr Wróbel, Piotr Kubisiak, Andrzej Eilmes*

Faculty of Chemistry, Jagiellonian University, Gronostajowa 2, 30-387 Kraków, Poland

Supporting Information

*e-mail: eilmes@chemia.uj.edu.pl 
Table S1. Compositions of the systems used in the simulations.

classical MD:

\begin{tabular}{lcccc}
\hline system & $\mathrm{N}_{\mathrm{Mg}}$ & $\mathrm{N}_{\text {TFSI }}$ & $\mathrm{N}_{\mathrm{Cl}}$ & $\mathrm{N}_{\mathrm{DME}}$ \\
\hline I & 61 & 82 & 40 & 500 \\
II, IIa & 86 & 86 & 86 & 500 \\
III, IIIa, IIIb, IIIc & 141 & 94 & 188 & 500 \\
\hline
\end{tabular}

initial structures:

systems I, II, III: $\mathrm{Mg}^{2+}$ and $\mathrm{Cl}^{-}$ions distributed randomly

system IIa: $\mathrm{Mg}^{2+}$ and $\mathrm{Cl}^{-}$ions as $\mathrm{Mg}_{2} \mathrm{Cl}_{2}^{2+}$

system IIIa: $\mathrm{Mg}^{2+}$ and $\mathrm{Cl}^{-}$ions as $\mathrm{MgCl}_{2}+\mathrm{Mg}_{2} \mathrm{Cl}_{2}^{2+}$

system IIIb: $\mathrm{Mg}^{2+}$ and $\mathrm{Cl}^{-}$ions as $\mathrm{Mg}_{3} \mathrm{Cl}_{4}^{2+}$ (I)

system IIIc: $\mathrm{Mg}^{2+}$ and $\mathrm{Cl}^{-}$ions as $\mathrm{Mg}_{3} \mathrm{Cl}_{4}^{2+}$ (II)

ab initio MD:

\begin{tabular}{lcccc}
\hline system & $\mathrm{N}_{\mathrm{Mg}}$ & $\mathrm{N}_{\mathrm{TFSI}}$ & $\mathrm{N}_{\mathrm{Cl}}$ & $\mathrm{N}_{\mathrm{DME}}$ \\
\hline III, IIIa, IIIb, IIIc & 3 & 2 & 4 & 30 \\
\hline
\end{tabular}

initial structures:

system III: $\mathrm{Mg}^{2+}$ and $\mathrm{Cl}^{-}$ions distributed randomly system IIIa: $\mathrm{Mg}^{2+}$ and $\mathrm{Cl}^{-}$ions as $\mathrm{MgCl}_{2}+\mathrm{Mg}_{2} \mathrm{Cl}_{2}^{2+}$

system IIIb: $\mathrm{Mg}^{2+}$ and $\mathrm{Cl}^{-}$ions as $\mathrm{Mg}_{3} \mathrm{Cl}_{4}^{2+}(\mathrm{I})$

system IIIc: $\mathrm{Mg}^{2+}$ and $\mathrm{Cl}^{-}$ions as $\mathrm{Mg}_{3} \mathrm{Cl}_{4}^{2+}$ (II) 
Table S2. Interatomic distances (in $\AA$ ) in QC-optimized structures. E stands for DME.

PBE/aug-cc-PVDZ

\begin{tabular}{|c|c|c|c|c|c|}
\hline & Mg-Mg & $\mathrm{Mg}-\mathrm{Cl}$ & $\mathrm{Cl}-\mathrm{Cl}$ & $\mathrm{Mg}-\mathrm{O}_{\mathrm{E}}$ & $\mathrm{Mg}-\mathrm{O}_{\mathrm{T}}$ \\
\hline $\mathrm{MgCl}^{+}$ & & 2.152 & & & \\
\hline $\mathrm{MgCl}_{2}$ & & $2 \times 2.206$ & 4.412 & & \\
\hline $\mathrm{MgCl}_{2} \cdot 2 \mathrm{E}$ & & $2.351,2.367$ & 3.910 & $\begin{array}{l}2.208,2.163 \\
2.337,2.339\end{array}$ & \\
\hline $\mathrm{Mg}_{2} \mathrm{Cl}_{2}^{2+}$ & 3.175 & $4 \times 2.358$ & 3.486 & & \\
\hline$\left[\mathrm{Mg}_{2} \mathrm{Cl}_{2} \cdot 4 \mathrm{E}\right]^{2+}$ & 3.534 & $\begin{array}{l}2.467,2.468, \\
2.476,2.479\end{array}$ & 3.456 & $\begin{array}{l}2.140,2.143, \\
2 \times 2.145, \\
2.146,2.147 \\
2.153,2.156\end{array}$ & \\
\hline $\mathrm{Mg}_{2} \mathrm{Cl}_{3}{ }^{+}$ & 2.681 & $6 \times 2.395$ & $3 \times 3.437$ & & \\
\hline $\mathrm{Mg}_{2} \mathrm{Cl}_{5}^{-}$ & 2.885 & $\begin{array}{l}2 \times 2.274, \\
6 \times 2.461\end{array}$ & $\begin{array}{l}3 \times 3.454, \\
6 \times 4.218\end{array}$ & & \\
\hline $\mathrm{Mg}_{3} \mathrm{Cl}_{4}{ }^{2+}(\mathrm{I})$ & $\begin{array}{l}2 \times 3.208, \\
6.415\end{array}$ & $\begin{array}{l}4 \times 2.298, \\
4 \times 2.452\end{array}$ & $\begin{array}{l}2 \times 3.500, \\
4 \times 4.234\end{array}$ & & \\
\hline $\mathrm{Mg}_{3} \mathrm{Cl}_{4}{ }^{2+}(\mathrm{II})$ & $\begin{array}{l}3.145 \\
2 \times 3.638\end{array}$ & $\begin{array}{l}2 \times 2.307 \\
4 \times 2.369 \\
2 \times 2.424 \\
2 \times 4.002 \\
2 \times 4.380\end{array}$ & $\begin{array}{l}3.486 \\
4 \times 3.836 \\
4.232\end{array}$ & & \\
\hline$\left[\mathrm{Mg}_{3} \mathrm{Cl}_{4} \cdot 5 \mathrm{E}\right]^{2+}$ & $\begin{array}{l}2 \times 3.542, \\
6.596\end{array}$ & $\begin{array}{l}2 \times(2.425, \\
2.454,2.489, \\
2.531)\end{array}$ & $\begin{array}{l}2 \times 3.445 \\
3.734 \\
2 \times 3.742 \\
5.057\end{array}$ & $\begin{array}{l}2 \times(2.145, \\
2.152,2.156, \\
2.180,2.182)\end{array}$ & \\
\hline $\begin{array}{l}{[\mathrm{MgTFSI}]^{+}} \\
\mathrm{MgTFSI}_{2}\end{array}$ & & & & & $\begin{array}{l}1.908,1.943 \\
4 \times 1.978\end{array}$ \\
\hline
\end{tabular}




\begin{tabular}{|c|c|c|c|c|c|}
\hline & $\mathrm{Mg}-\mathrm{Mg}$ & $\mathrm{Mg}-\mathrm{Cl}$ & $\mathrm{Cl}-\mathrm{Cl}$ & $\mathrm{Mg}-\mathrm{O}_{\mathrm{E}}$ & $\mathrm{Mg}-\mathrm{O}_{\mathrm{T}}$ \\
\hline $\mathrm{MgCl}^{+}$ & & 2.262 & & & \\
\hline $\mathrm{MgCl}_{2}$ & & $2 \times 2.292$ & 4.583 & & \\
\hline $\mathrm{MgCl}_{2} \cdot 2 \mathrm{E}$ & & $2.349,2.365$ & 3.878 & $\begin{array}{l}2.141,2.180 \\
2.300,2.309\end{array}$ & \\
\hline $\mathrm{Mg}_{2} \mathrm{Cl}_{2}^{2+}$ & 3.382 & $4 \times 2.434$ & 3.494 & & \\
\hline$\left[\mathrm{Mg}_{2} \mathrm{Cl}_{2} \cdot 4 \mathrm{E}\right]^{2+}$ & 3.539 & $\begin{array}{l}2.473,2.479 \\
2.480,2.488\end{array}$ & 3.447 & $\begin{array}{l}2.123,2.129, \\
2.141,2.142, \\
2.145, \\
2 \times 2.152, \\
2.169\end{array}$ & \\
\hline $\mathrm{Mg}_{2} \mathrm{Cl}_{3}^{+}$ & 2.908 & $\begin{array}{l}2 \times(2.450 \\
2.455,2.466)\end{array}$ & $\begin{array}{l}3.406,3.439 \\
3.441\end{array}$ & & \\
\hline $\mathrm{Mg}_{2} \mathrm{Cl}_{5}^{-}$ & 2.995 & $\begin{array}{l}2 \times 2.322 \\
4 \times 2.491 \\
2 \times 3.495\end{array}$ & $\begin{array}{l}3.433,3.447, \\
3.472, \\
2 \times(4.294, \\
4.313,4.316)\end{array}$ & & \\
\hline $\mathrm{Mg}_{3} \mathrm{Cl}_{4}{ }^{2+}(\mathrm{I})$ & $\begin{array}{l}2 \times 3.331 \\
6.663\end{array}$ & $\begin{array}{l}4 \times 2.414 \\
4 \times 2.443\end{array}$ & $\begin{array}{l}2 \times 3.534 \\
4 \times 4.199\end{array}$ & & \\
\hline $\mathrm{Mg}_{3} \mathrm{Cl}_{4}{ }^{2+}(\mathrm{II})$ & $\begin{array}{l}3.368, \\
3.705 \\
3.727\end{array}$ & $\begin{array}{l}2.388,2.396, \\
2 \times 2.430, \\
2 \times 2.436, \\
2.456,2.463, \\
3.819,3.822, \\
4.561,4.594\end{array}$ & $\begin{array}{l}3.508, \\
2 \times 3.751, \\
3.769,3.770 \\
4.424\end{array}$ & & \\
\hline$\left[\mathrm{Mg}_{3} \mathrm{Cl}_{4} \cdot 5 \mathrm{E}\right]^{2+}$ & $\begin{array}{l}2 \times 3.526 \\
6.578\end{array}$ & $\begin{array}{l}2 \times(2.433 \\
2.462,2.486 \\
2.530)\end{array}$ & $\begin{array}{l}2 \times 3.462 \\
2 \times 3.682 \\
3.738,5.058\end{array}$ & $\begin{array}{l}2 \times(2.138, \\
2.146,2.149, \\
2.168,2.173)\end{array}$ & \\
\hline $\begin{array}{l}{\left[\mathrm{MgTFSI}^{+}\right.} \\
\mathrm{MgTFSI}_{2}\end{array}$ & & & & & $\begin{array}{l}1.998,2.006 \\
2 \times 2.042, \\
2 \times 2.043\end{array}$ \\
\hline
\end{tabular}


B3LYP/aug-cc-pVDZ

vacuum

\begin{tabular}{|c|c|c|c|c|c|}
\hline & Mg-Mg & $\mathrm{Mg}-\mathrm{Cl}$ & $\mathrm{Cl}-\mathrm{Cl}$ & $\mathrm{Mg}-\mathrm{O}_{\mathrm{E}}$ & $\mathrm{Mg}-\mathrm{O}_{\mathrm{T}}$ \\
\hline $\mathrm{MgCl}^{+}$ & & 2.143 & & & \\
\hline $\mathrm{MgCl}_{2}$ & & $2 \times 2.198$ & 4.396 & & \\
\hline $\mathrm{MgCl}_{2} \cdot 2 \mathrm{E}$ & & $2.349,2.365$ & 3.878 & $\begin{array}{l}2.141,2.180 \\
2.300,2.309\end{array}$ & \\
\hline $\mathrm{Mg}_{2} \mathrm{Cl}_{2}{ }^{2+}$ & 3.184 & $4 \times 2.352$ & 3.462 & & \\
\hline$\left[\mathrm{Mg}_{2} \mathrm{Cl}_{2} \cdot 4 \mathrm{E}\right]^{2+}$ & 3.568 & $\begin{array}{l}2.482,2.486, \\
2.458,2.465\end{array}$ & 3.403 & $\begin{array}{l}2 \times 2.100, \\
2.114,2.119, \\
2.125,2.134, \\
2.141,2.149\end{array}$ & \\
\hline $\mathrm{Mg}_{2} \mathrm{Cl}_{3}{ }^{+}$ & 2.682 & $6 \times 2.389$ & $3 \times 3.426$ & & \\
\hline $\mathrm{Mg}_{2} \mathrm{Cl}_{5}^{-}$ & 2.887 & $\begin{array}{l}2 \times 2.265 \\
6 \times 2.458\end{array}$ & $\begin{array}{l}3 \times 3.445 \\
6 \times 4.208\end{array}$ & & \\
\hline $\mathrm{Mg}_{3} \mathrm{Cl}_{4}{ }^{2+}(\mathrm{I})$ & $\begin{array}{l}2 \times 3.216 \\
6.432\end{array}$ & $\begin{array}{l}4 \times 2.292 \\
4 \times 2.446\end{array}$ & $\begin{array}{l}2 \times 3.476, \\
4 \times 4.231\end{array}$ & & \\
\hline $\mathrm{Mg}_{3} \mathrm{Cl}_{4}{ }^{2+}(\mathrm{II})$ & $\begin{array}{l}3.143, \\
3.698, \\
3.699\end{array}$ & $\begin{array}{l}2 \times 2.299 \\
4 \times 2.362, \\
2 \times 2.420 \\
2 \times 4.068 \\
4.350,4.351\end{array}$ & $\begin{array}{l}3.463, \\
4 \times 3.822, \\
4.158\end{array}$ & & \\
\hline$\left[\mathrm{Mg}_{3} \mathrm{Cl}_{4} \cdot 5 \mathrm{E}\right]^{2+}$ & $\begin{array}{l}2 \times 3.534 \\
6.577\end{array}$ & $\begin{array}{l}2 \times(2.425, \\
2.454,2.484, \\
2.530)\end{array}$ & $\begin{array}{l}2 \times 3.448, \\
3.691, \\
2 \times 3.746, \\
5.054\end{array}$ & $\begin{array}{l}2 \times(2.118, \\
2.124,2.126, \\
2.146,2.152)\end{array}$ & \\
\hline [MgTFSI $^{+}$ & & & & & $1.893,1.927$ \\
\hline $\mathrm{MgTFSI}_{2}$ & & & & & $4 \times 1.960$ \\
\hline
\end{tabular}




\begin{tabular}{|c|c|c|c|c|c|}
\hline & $\mathrm{Mg}-\mathrm{Mg}$ & $\mathrm{Mg}-\mathrm{Cl}$ & $\mathrm{Cl}-\mathrm{Cl}$ & $\mathrm{Mg}-\mathrm{O}_{\mathrm{E}}$ & $\mathrm{Mg}-\mathrm{O}_{\mathrm{T}}$ \\
\hline $\mathrm{MgCl}^{+}$ & & 2.256 & & & \\
\hline $\mathrm{MgCl}_{2}$ & & $2 \times 2.284$ & 4.569 & & \\
\hline $\mathrm{MgCl}_{2} \cdot 2 \mathrm{E}$ & & $2.401,2.421$ & 3.765 & $\begin{array}{l}2.126,2.152 \\
2.219,2.225\end{array}$ & \\
\hline $\mathrm{Mg}_{2} \mathrm{Cl}_{2}{ }^{2+}$ & 3.385 & $\begin{array}{l}2.432 \\
2 \times 2.433 \\
2.434\end{array}$ & 3.488 & & \\
\hline$\left[\mathrm{Mg}_{2} \mathrm{Cl}_{2} \cdot 4 \mathrm{E}\right]^{2+}$ & 3.532 & $\begin{array}{l}2.472,2.479 \\
2.481,2.490\end{array}$ & 3.447 & $\begin{array}{l}2.103,2.104, \\
2.109,2.112, \\
2.118,2.123, \\
2.127,2.134\end{array}$ & \\
\hline $\mathrm{Mg}_{2} \mathrm{Cl}_{3}^{+}$ & 2.927 & $\begin{array}{l}2.456,2.461, \\
2.462,2.463, \\
2.464,2.473\end{array}$ & $\begin{array}{l}3.413,3.426, \\
3.455, \\
2 \times 4.289, \\
2 \times 4.308, \\
4.311,4.312\end{array}$ & & \\
\hline $\mathrm{Mg}_{2} \mathrm{Cl}_{5}^{-}$ & 3.003 & $\begin{array}{l}3.428,3.441 \\
3.465\end{array}$ & $\begin{array}{l}2 \times 2.314 \\
3 \times 2.490 \\
2.491 \\
2 \times 2.495\end{array}$ & & \\
\hline $\mathrm{Mg}_{3} \mathrm{Cl}_{4}{ }^{2+}(\mathrm{I})$ & $\begin{array}{l}2 \times 3.336, \\
6.672\end{array}$ & $\begin{array}{l}4 \times 2.411 \\
4 \times 2.441\end{array}$ & $\begin{array}{l}2 \times 3.523 \\
4 \times 4.198\end{array}$ & & \\
\hline $\mathrm{Mg}_{3} \mathrm{Cl}_{4}{ }^{2+}$ (II) & $\begin{array}{l}3.377 \\
2 \times 3.818\end{array}$ & $\begin{array}{l}2 \times(2.387, \\
2.432,2.433, \\
2.460), \\
3.964,3.966, \\
2 \times 4.545\end{array}$ & $\begin{array}{l}3.491 \\
2 \times 3.771 \\
2 \times 3.772, \\
4.326\end{array}$ & & \\
\hline$\left[\mathrm{Mg}_{3} \mathrm{Cl}_{4} \cdot 5 \mathrm{E}\right]^{2+}$ & $\begin{array}{l}2 \times 3.520 \\
7.834\end{array}$ & $\begin{array}{l}2 \times(2.432, \\
2.463,2.482, \\
2.531)\end{array}$ & $\begin{array}{l}2 \times 3.467 \\
3.690 \\
2 \times 3.701 \\
5.060\end{array}$ & $\begin{array}{l}2 \times(2.110, \\
2.117,2.118, \\
2.137,2.141)\end{array}$ & \\
\hline $\begin{array}{l}{\left[\mathrm{MgTFSI}^{+}\right.} \\
\mathrm{MgTFSI}_{2}\end{array}$ & & & & & $\begin{array}{l}1.985,1.988 \\
1.989,1.992, \\
2.001,2.003\end{array}$ \\
\hline
\end{tabular}


Definition of the total binding effect presented in Table 2 in the main paper.

For the system containing $n$ complexes $\mathrm{Mg}_{x n} \mathrm{Cl}_{y n}$ and $m$ complexes $\mathrm{Mg}_{x m} \mathrm{Cl}_{y m}$ the net binding effect $E_{\mathrm{B}}$ in vacuum or in the PCM solvent is defined as:

$$
E_{\mathrm{B}}=n \cdot E\left(\mathrm{Mg}_{x n} \mathrm{Cl}_{y n}\right)+m \cdot E\left(\mathrm{Mg}_{x m} \mathrm{Cl}_{y m}\right)-(n \cdot x n+m \cdot x m) \cdot E\left(\mathrm{Mg}^{2+}\right)-(n \cdot y n+m \cdot y m) \cdot E\left(\mathrm{Cl}^{-}\right),
$$

where $E(Z)$ stands for the energy of species $\mathrm{Z}$ in vacuum or in the PCM, respectively.

For example, $E_{\mathrm{B}}$ for $2 \mathrm{MgCl}^{+}+\mathrm{MgCl}_{2}$ equals

$$
E_{\mathrm{B}}=2 E\left(\mathrm{MgCl}^{+}\right)+E\left(\mathrm{MgCl}_{2}\right)-3 E\left(\mathrm{Mg}^{2+}\right)-4 E\left(\mathrm{Cl}^{-}\right)
$$


FF-MD
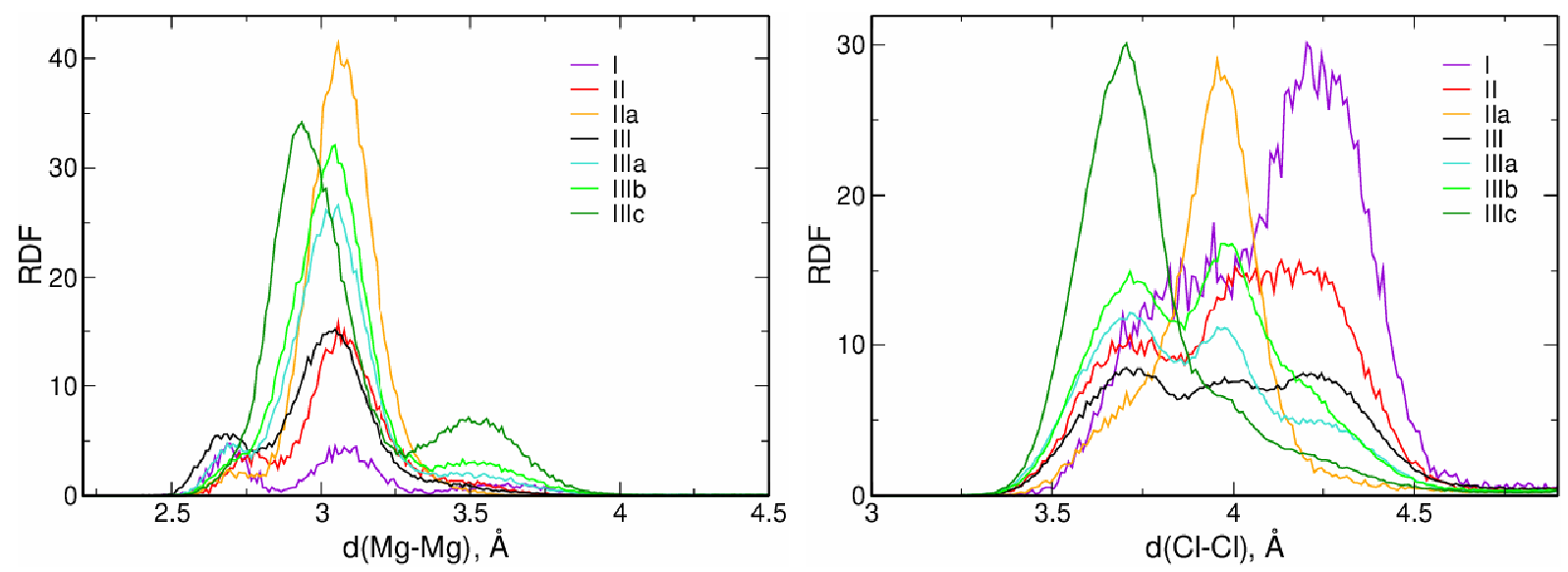

AIMD
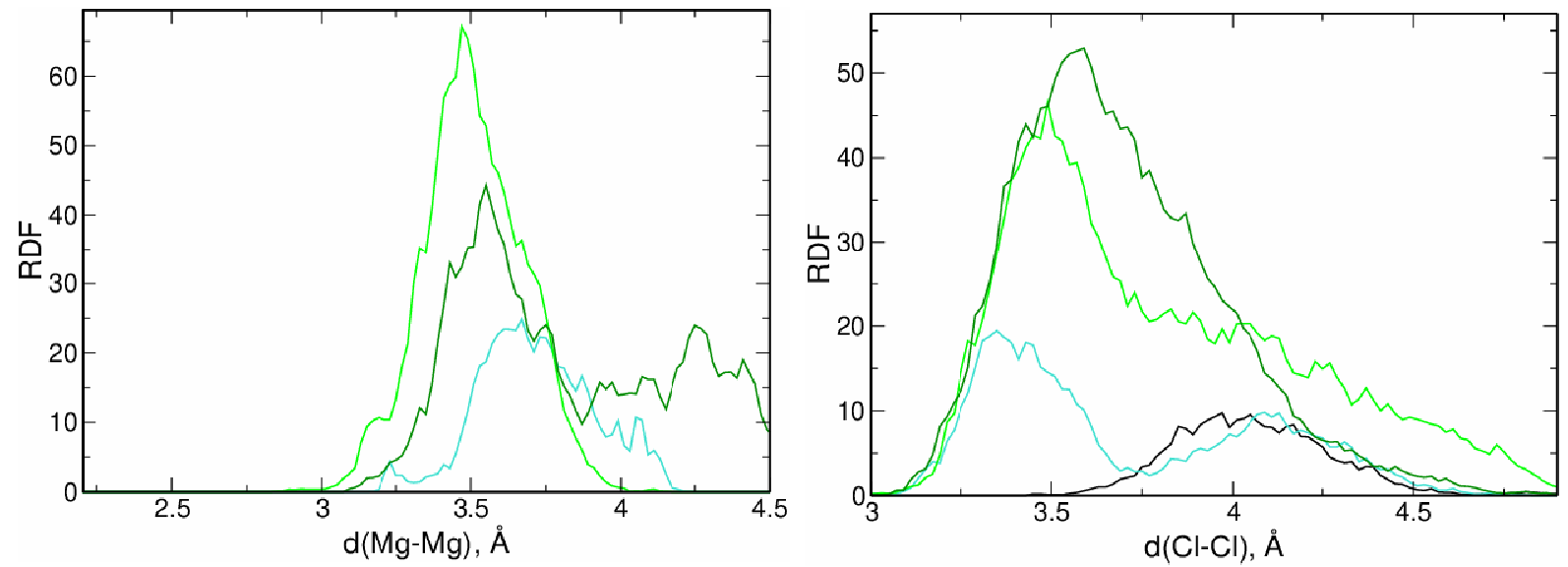

Figure S1. RDFs for $\mathrm{Mg}-\mathrm{Mg}$ and $\mathrm{Cl}-\mathrm{Cl}$ pairs obtained from classical (top) and ab initio (bottom) MD simulations. 


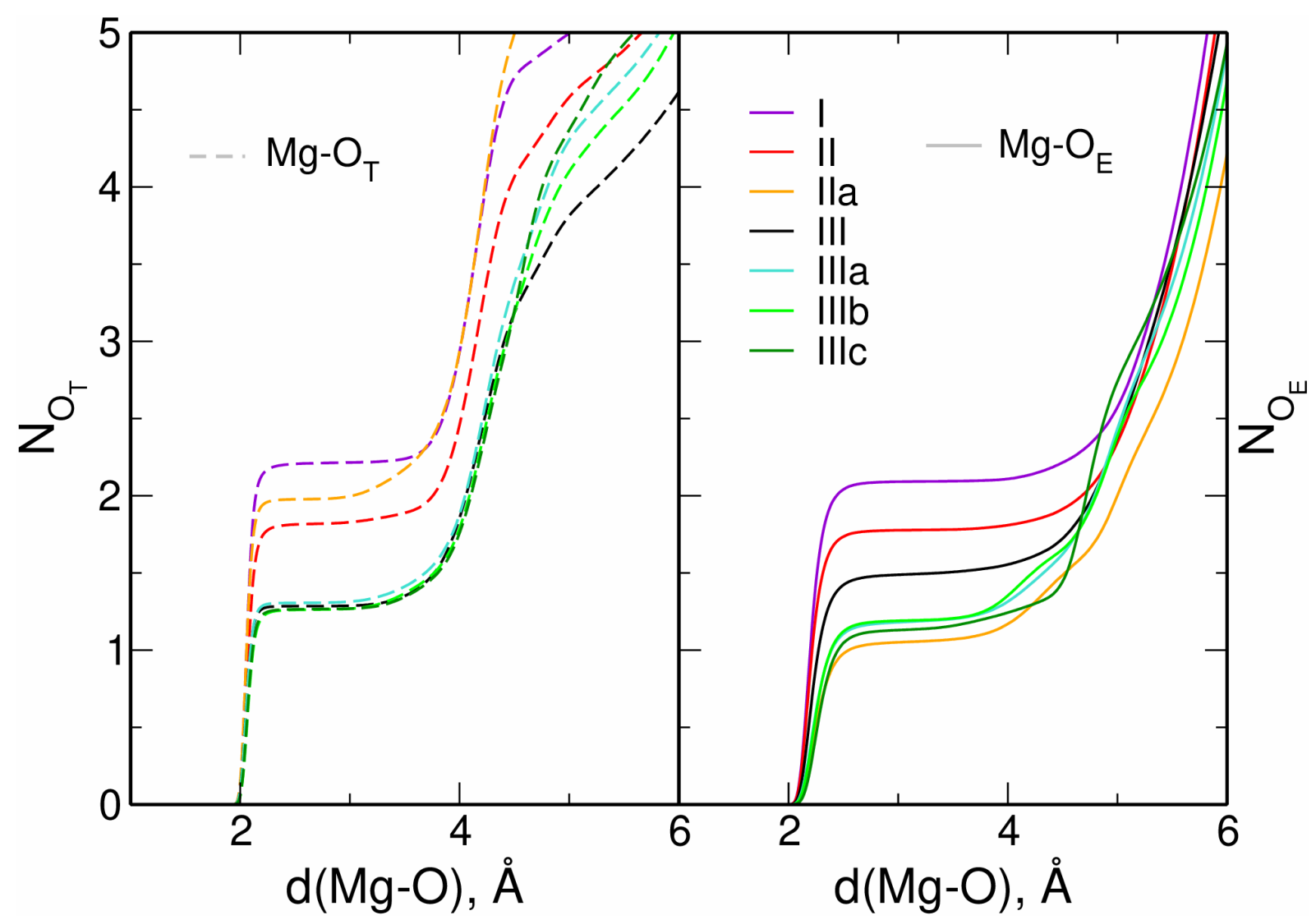

Figure S2. Integrated RDFs (running coordination numbers) for Mg-O pairs obtained from classical simulations. Oxygen atoms from TFSI anions and DME molecules are marked as $\mathrm{O}_{\mathrm{T}}$ and $\mathrm{O}_{\mathrm{E}}$, respectively. 

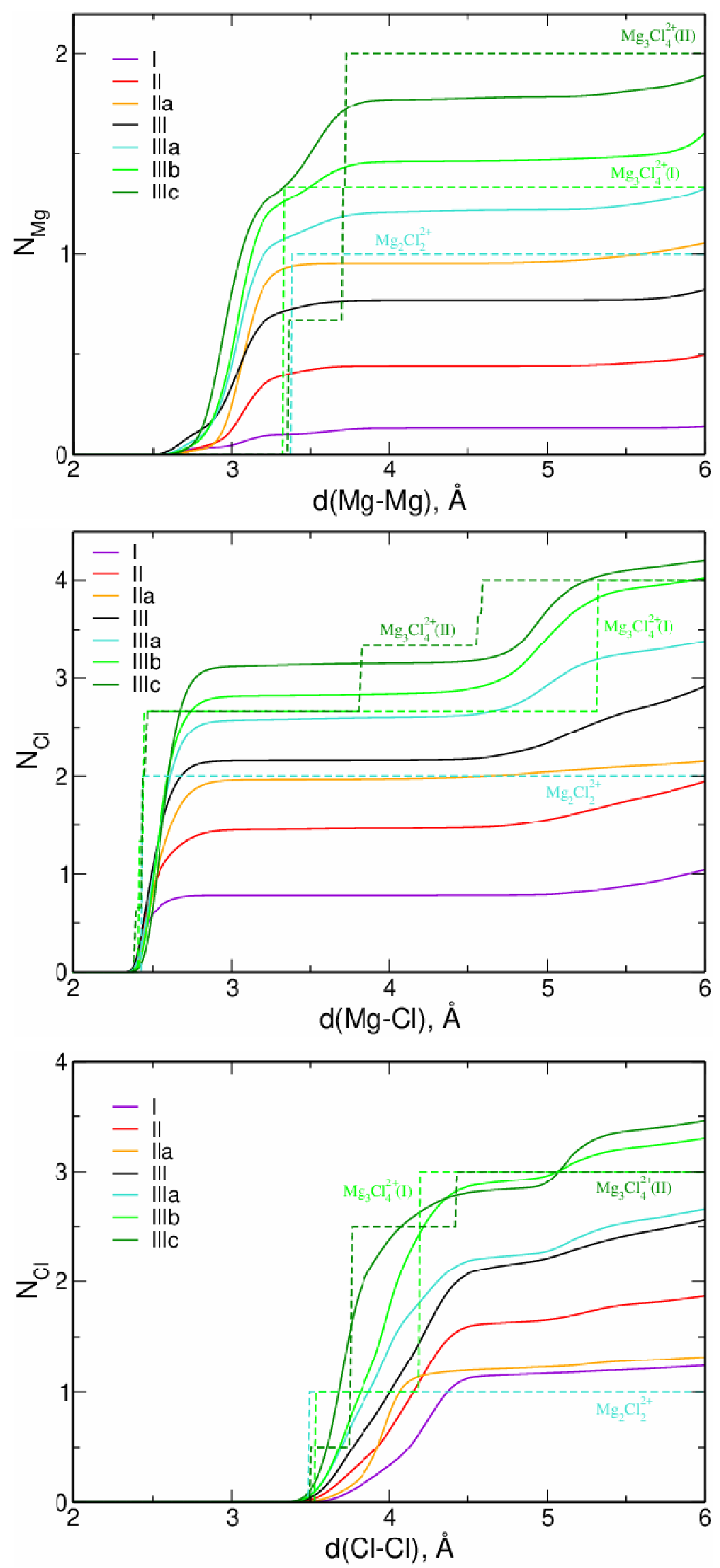

Figure S3. Integrated RDFs for systems studied in FF-MD simulations. Broken lines mark the corresponding “ideal” RDFs for geometries of aggregates optimized in QC calculations. 

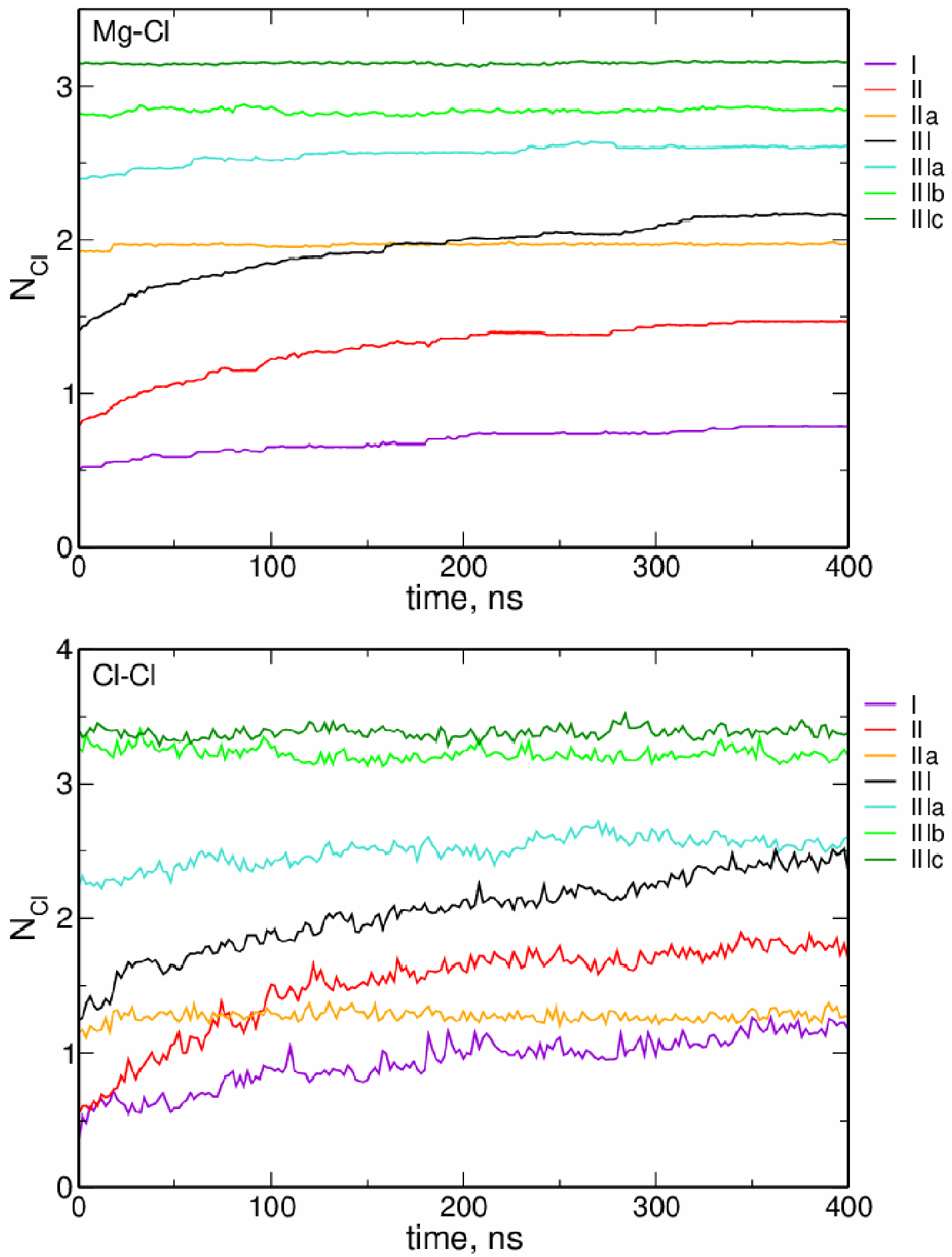

Figure S4. Changes in the average number of $\mathrm{Cl}^{-}$ions found at the distance $4 \AA$ from the $\mathrm{Mg}$ cation or at the distance $5.5 \AA$ from the $\mathrm{Cl}$ anion. 

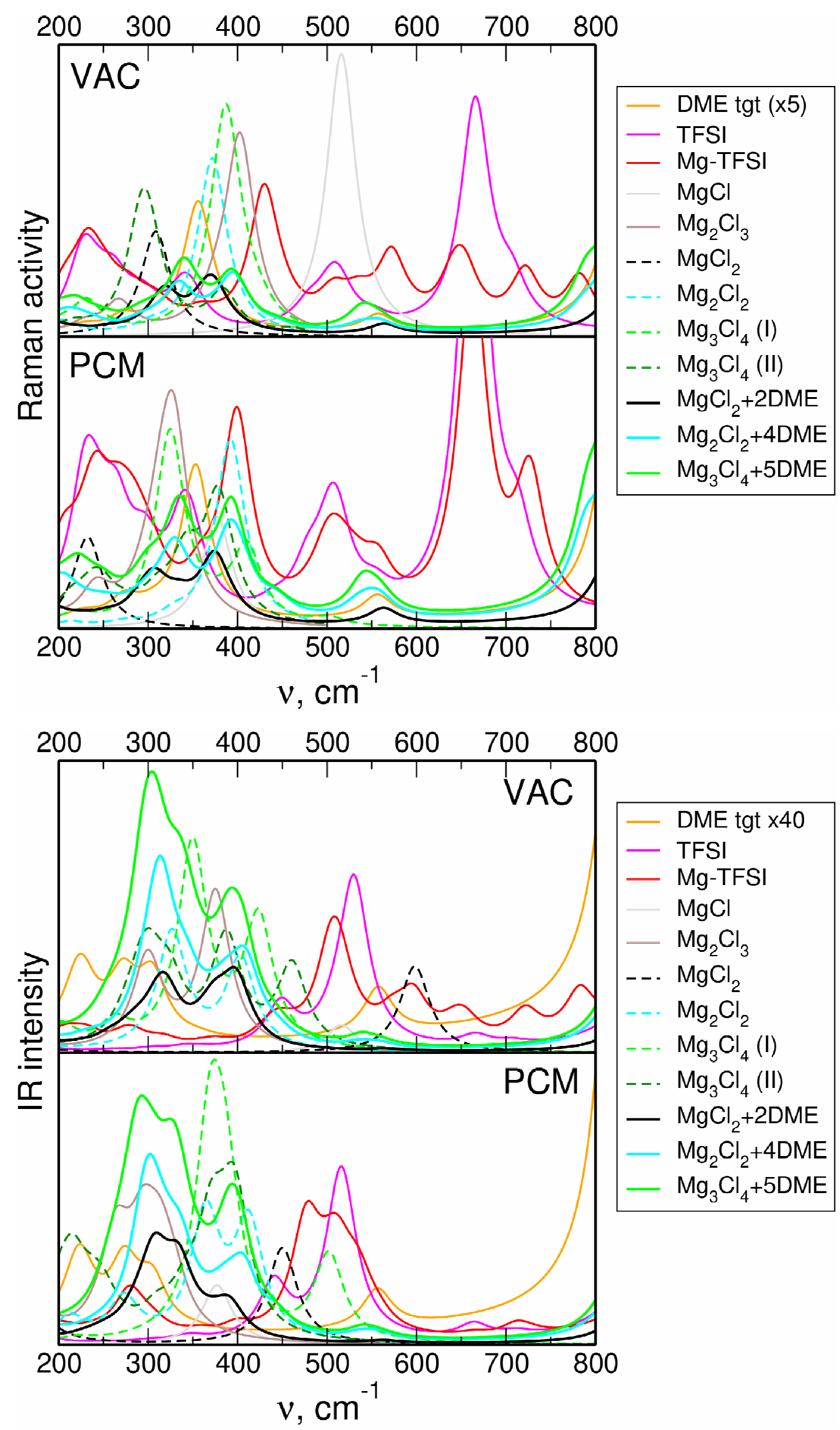

Figure S5. Raman and IR spectra calculated at the PBE/aug-cc-pVDZ level. 
Table S3. Comparison between the experimental data from the Supporting Information of $J$. Phys. Chem. C 2017, 121, 24909-24918 and the frequencies of the vibrations with largest Raman activities calculated for isolated complexes at the PBE/aug-cc-pVDZ level in the PCM solvent. Results from FT analysis of interatomic distances in AIMD simulations are included in the last column. Frequencies in $\mathrm{cm}^{-1}$.

Experimental data for $\mathrm{MgCl}_{2}$ from DME, QC data for $\mathrm{MgCl}_{2}$ and $\mathrm{MgCl}_{2} \cdot 2 \mathrm{DME}, \mathrm{FT} / \mathrm{AIMD}$ for system IIIa:

\begin{tabular}{|c|c|c|c|c|}
\hline \multicolumn{2}{|c|}{ Experiment } & \multicolumn{2}{|c|}{ PBE/aug-cc-pVDZ } & FT from AIMD \\
\hline frequency & assignment & $\mathrm{MgCl}_{2}$ & $\mathrm{MgCl}_{2} \cdot 2 \mathrm{DME}$ & \\
\hline 184 & na & & 183-194 & \\
\hline 218 & $\mathrm{Mg}-\mathrm{Cl}$ vibr. & 232 & & $220-240 \mathrm{Mg}-\mathrm{Cl}$ \\
\hline 306 & na & & 307 & \\
\hline 334 & na & & 334 & $\mathrm{Mg}-\mathrm{Mg}$ \\
\hline 390 & na & & 375 & \\
\hline & & & 564 & \\
\hline 875 & DME & & 855 & \\
\hline & & & 1006 & \\
\hline
\end{tabular}

Experimental data for $\mathrm{Mg}_{2} \mathrm{Cl}_{2}$ (DME) $)_{4} \cdot 2 \mathrm{TFSI}$ solvate, QC data for $\mathrm{Mg}_{2} \mathrm{Cl}_{2}$ and $\mathrm{Mg}_{2} \mathrm{Cl}_{2} \cdot 4 \mathrm{DME}$, FT/AIMD for system IIIa:

\begin{tabular}{|c|c|c|c|c|}
\hline \multicolumn{2}{|c|}{ Experiment } & \multicolumn{2}{|c|}{ PBE/aug-cc-pVDZ } & FT from AIMD \\
\hline frequency & assignment & $\mathrm{Mg}_{2} \mathrm{Cl}_{2}$ & $\mathrm{Mg}_{2} \mathrm{Cl}_{2} \cdot 4 \mathrm{DME}$ & \\
\hline 220 & $\mathrm{Mg}-\mathrm{Cl}$ vibr. & 212 v.w. & $203,208,210$ & $150,200 \mathrm{Mg}-\mathrm{Cl}$ \\
\hline 340 & TFSI & & 330 & \\
\hline 398 & TFSI & 392 & 392 & \\
\hline 551 & DME & & 534,549 & \\
\hline 570 & DME & & 560 & \\
\hline 880 & solv. cage & & $841,850,854$ & \\
\hline 1018 & DME & & $1000-1020$ & \\
\hline 1095 & DME & & $1072-1084$ & \\
\hline 1140 & DME,TFSI & & $1135-1137$ & \\
\hline 1244 & TFSI & & $1213-1222$ & \\
\hline 1284 & DME & & $1247-1274$ & \\
\hline $1450-1490$ & DME & & $1420-1455$ & \\
\hline
\end{tabular}


Experimental data for $\mathrm{Mg}_{3} \mathrm{Cl}_{4}(\mathrm{DME})_{5} \cdot 2 \mathrm{TFSI}$ solvate, QC data for $\mathrm{Mg}_{3} \mathrm{Cl}_{4}$ (I) and $\mathrm{Mg}_{3} \mathrm{Cl}_{4} \cdot 5 \mathrm{DME}, \mathrm{FT} / \mathrm{AIMD}$ for system IIIb:

\begin{tabular}{|c|c|c|c|c|}
\hline \multicolumn{2}{|c|}{ Experiment } & \multicolumn{2}{|c|}{ PBE/aug-cc-pVDZ } & FT from AIMD \\
\hline frequency & assignment & $\mathrm{Mg}_{3} \mathrm{Cl}_{4}$ & $\mathrm{Mg}_{3} \mathrm{Cl}_{4} \cdot 5 \mathrm{DME}$ & \\
\hline 174 & na & 174 & 191,197 & $150 \mathrm{Mg}-\mathrm{Cl}$ \\
\hline 220 & Mg-Cl vibr. & 220 & 220 & $200-240 \mathrm{Mg}-\mathrm{Cl}$ \\
\hline 326 & TFSI & 325 & 335 & \\
\hline 410 & TFSI & 411 & 393 & \\
\hline 550 & DME & & 540 & \\
\hline 880 & solv. cage & & 849 & \\
\hline 1017 & DME & & 992,1000 & \\
\hline 1097 & DME & & 1082,1087 & \\
\hline 1138 & DME,TFSI & & $1128,1134,1139$ & \\
\hline 1242 & TFSI & & 1249-1252 & \\
\hline 1284 & DME & & 1265 & \\
\hline 1450 & DME & & $1410-1444$ & \\
\hline 1460 & & & & \\
\hline 1475 & & & & \\
\hline
\end{tabular}



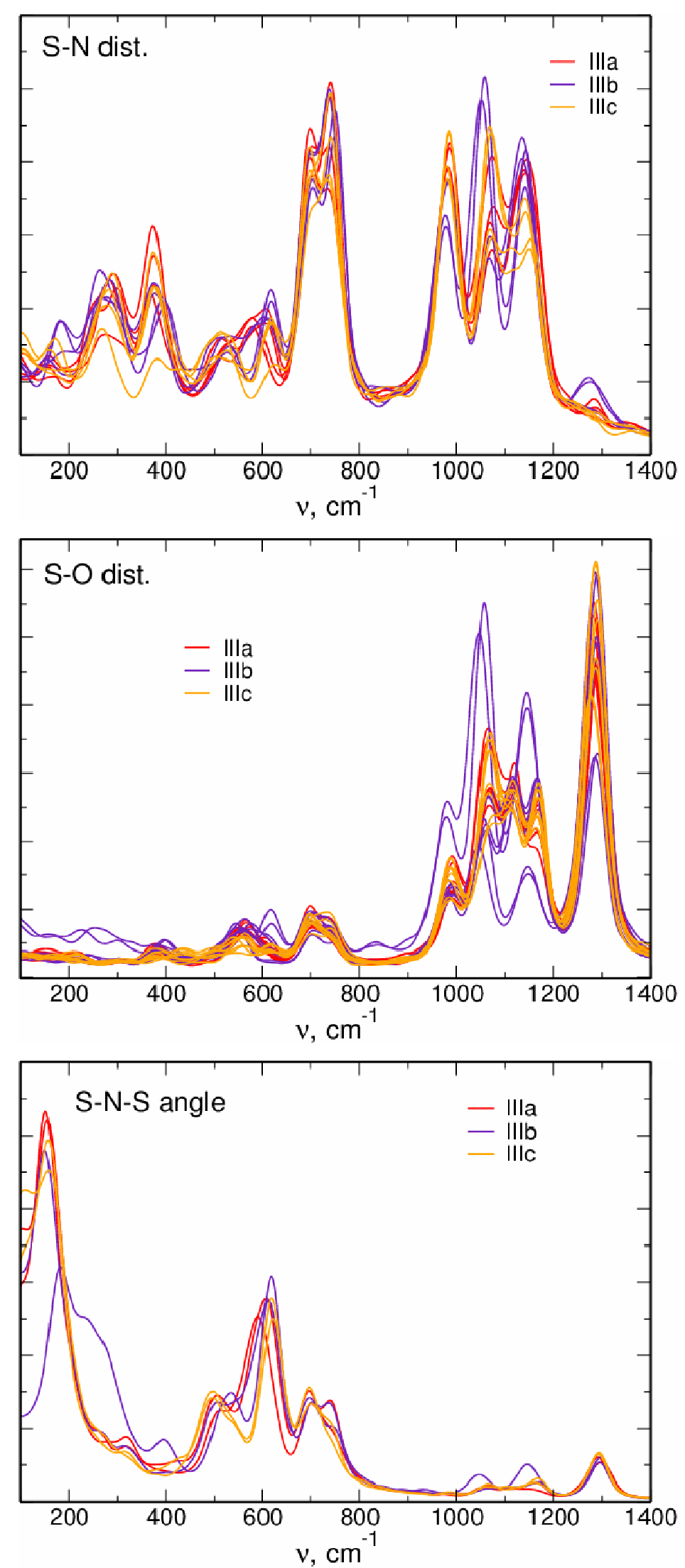

Figure S6. FTs of geometric parameters of TFSI anions obtained from the AIMD simulations. 
Force field parameters

$$
\begin{aligned}
& U_{i j}=\sum_{i} K_{r, i}\left(r_{i}-r_{0, i}\right)^{2}+\sum_{i} K_{\theta, i}\left(\theta_{i}-\theta_{0, i}\right)^{2}+\sum_{n} K_{\xi, n}(1+\cos (n(\xi)-\gamma)) \\
& +\sum_{i} \sum_{j \neq i}\left\{\varepsilon_{i j}\left(\frac{r_{m i n, i j}}{r_{i j}}\right)^{12}-2\left(\frac{r_{m i n, i j}}{r_{i j}}\right)^{6}+\frac{q_{i} q_{j}}{4 \pi \varepsilon_{0} r_{i j}}\right\} \\
& \varepsilon_{i j}=\sqrt{\varepsilon_{i} \varepsilon_{j}} \\
& r_{\text {min }, i j}=\left(r_{\text {min }, i}+r_{\text {min }, j}\right) / 2
\end{aligned}
$$

In the force field, Drude particle with partial charge $q_{\mathrm{D}, i}$ is attached to atom with polarizability $\alpha_{i}$ :

$\alpha_{i}=\frac{q_{D, i}^{2}}{2 k_{D}}$

Partial charge on the parent atom is modified, so that the atom and the Drude particle together carry the original partial charge $q$ of the parent atom.

\section{TFSI anion}

BONDS $\begin{gathered}\mathrm{K}_{\mathrm{r}} \\ {\left[\mathrm{kcal} / \mathrm{mole} / \AA^{2}\right]}\end{gathered} \quad \mathrm{r}_{0}[\AA]$

\begin{tabular}{ccc}
\hline Ct-Ft & 441.8 & 1.323 \\
Ct-St & 235.4 & 1.818 \\
St-Ot & 637.1 & 1.442 \\
St-Nt & 372.0 & 1.570 \\
\hline \multicolumn{3}{c}{} \\
ANGLES & $\mathrm{K}_{\theta}$ & \\
& {$\left[\mathrm{kcal} / \mathrm{mole} \mathrm{rad}^{2}\right]$} & $\theta[\mathrm{deg}]$ \\
\hline Ft-Ct-Ft & 93.3 & 107.1 \\
Ft-Ct-St & 82.9 & 111.8 \\
Ct-St-Ot & 104.0 & 102.6 \\
Ot-St-Ot & 115.8 & 118.5 \\
Os-St-Nt & 94.3 & 113.6 \\
Ct-Sn-Nt & 97.5 & 100.2 \\
St-Nt-St & 80.2 & 125.6 \\
\hline
\end{tabular}

DIHEDRALS $\begin{gathered}\mathrm{K}_{\xi, \mathrm{n}} \\ {[\mathrm{kcal} / \mathrm{mole}]}\end{gathered} \quad \mathrm{n} \quad \gamma[\mathrm{deg}]$

\begin{tabular}{cccc}
\hline Ot-St-Ct-Ft & 0.173 & 3 & 0.0 \\
Nt-St-Ct-Ft & 0.158 & 3 & 0.0 \\
Ot-St-Nt-St & -0.0018 & 3 & 0.0 \\
Ct-St-Nt-St & 3.916 & 1 & 0.0 \\
Ct-St-Nt-St & -1.245 & 2 & 180.0 \\
Ct-St-Nt-St & -0.382 & 3 & 0.0 \\
\hline
\end{tabular}




\begin{tabular}{|c|c|c|}
\hline VDW & $\begin{array}{c}\varepsilon \\
{[\mathrm{kcal} / \mathrm{mole}]}\end{array}$ & $\mathrm{r}_{\min }[\AA]$ \\
\hline $\mathrm{Nt}$ & 0.0510 & 1.824 \\
\hline $\mathrm{St}$ & 0.0750 & 2.291 \\
\hline Ot & 0.0630 & 1.944 \\
\hline $\mathrm{Ct}$ & 0.0200 & 1.768 \\
\hline $\mathrm{Ft}$ & 0.0160 & 1.490 \\
\hline $\begin{array}{c}\text { ELECTRO- } \\
\text { STATIC }\end{array}$ & $\mathrm{q}[\mathrm{e}]$ & $\alpha\left[\AA^{3}\right]$ \\
\hline $\mathrm{Nt}$ & -0.4854 & 1.45 \\
\hline $\mathrm{St}$ & 0.5928 & 0.50 \\
\hline Ot & -0.3893 & 1.36 \\
\hline $\mathrm{Ct}$ & 0.1391 & 1.05 \\
\hline $\mathrm{Ft}$ & -0.0702 & 0.60 \\
\hline
\end{tabular}

\section{DME molecule}

\begin{tabular}{lcc}
\hline \multicolumn{3}{c}{$\mathrm{K}_{\mathrm{r}}$} \\
BONDS & {$\left[\mathrm{kcal} / \mathrm{mole} / \AA^{2}\right]$} & $\mathrm{r}_{0}[\AA]$ \\
\hline C2D-C2D & 268.0 & 1.529 \\
C2D-HD & 340.0 & 1.090 \\
C2D-OS & 320.0 & 1.410 \\
C3D-OD & 320.0 & 1.410 \\
C3D-HD & 340.0 & 1.090 \\
\hline \multicolumn{4}{c}{} \\
ANGLES & {$\left[\mathrm{kcal} / \mathrm{mole}_{\theta} \mathrm{rad}^{2}\right]$} \\
\multicolumn{3}{c}{$\theta[\mathrm{deg}]$} \\
\hline C2D-C2D-HD & 37.5 & 110.7 \\
HD-C2D-HD & 33.0 & 107.8 \\
C2D-C2D-OS & 50.0 & 109.5 \\
OS-C2D-HD & 35.0 & 109.5 \\
OS-C3D-HD & 35.0 & 109.5 \\
HD-C3D-HD & 33.0 & 107.8 \\
C3D-OS-C2D & 60.0 & 109.5 \\
\hline
\end{tabular}




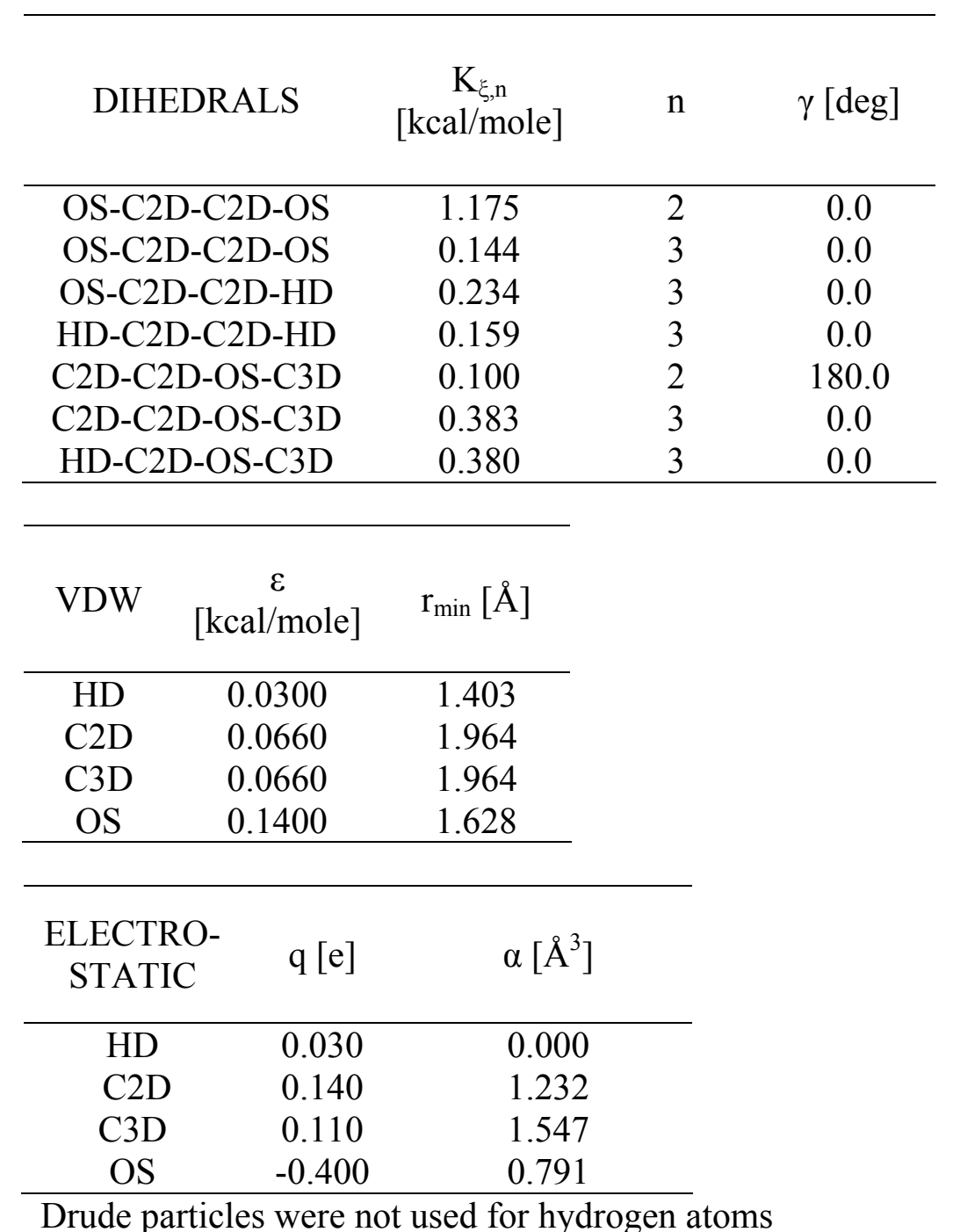

Drude particles were not used for hydrogen atoms 


\begin{tabular}{|c|c|c|}
\hline \multicolumn{3}{|l|}{$\mathrm{Mg}^{2+}$} \\
\hline VDW & $\begin{array}{c}\varepsilon \\
{[\mathrm{kcal} / \mathrm{mole}]}\end{array}$ & $\mathrm{r}_{\min }[\AA]$ \\
\hline $\mathrm{Mg}$ & 0.2000 & 1.470 \\
\hline $\begin{array}{l}\text { VDW- } \\
\text { pairs }\end{array}$ & $\begin{array}{c}\varepsilon \\
{[\mathrm{kcal} / \mathrm{mole}]}\end{array}$ & $\mathrm{r}_{\min }[\AA]$ \\
\hline $\mathrm{Mg}-\mathrm{Nt}$ & 0.0714 & 3.400 \\
\hline $\mathrm{Mg}-\mathrm{Ft}$ & 0.0400 & 3.150 \\
\hline $\mathrm{Mg}-\mathrm{Ot}$ & 0.0794 & 3.250 \\
\hline \multicolumn{2}{|c|}{$\begin{array}{l}\text { ELECTRO- } \\
\text { STATIC }\end{array}$} & \\
\hline $\mathrm{Mg}$ & 2.000 & \\
\hline \multicolumn{3}{|c|}{ Drude particles were not used for $\mathrm{Mg}^{2+}$ cation } \\
\hline VDW & $\begin{array}{c}\varepsilon \\
{[\mathrm{kcal} / \mathrm{mole}]}\end{array}$ & $\mathrm{r}_{\min }[\AA]$ \\
\hline $\mathrm{Cl}$ & 0.3400 & 2.150 \\
\hline $\begin{array}{r}\text { ELECTR } \\
\text { STATI }\end{array}$ & $\mathrm{q}[\mathrm{e}]$ & $\alpha\left[\AA^{3}\right]$ \\
\hline $\mathrm{Cl}$ & -1.000 & 4.000 \\
\hline
\end{tabular}

